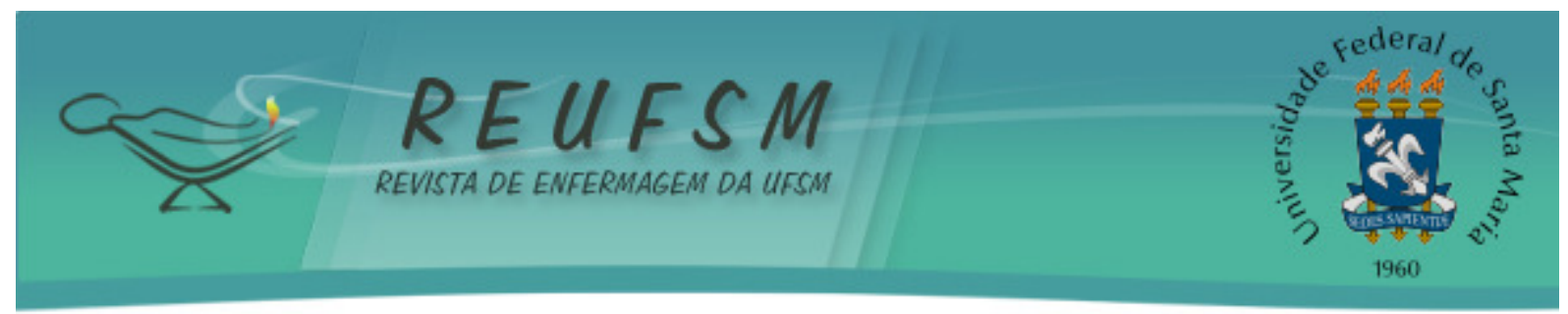

ARTIGO DE REVISÃO

\title{
CUIDADOS DE ENFERMAGEM À CRIANÇA COM CÂNCER: UMA REVISÃO INTEGRATIVA DA LITERATURA
}

\author{
NURSING CARE TO THE CHILD WITH CANCER: AN INTEGRATIVE REVIEW OF THE \\ LITERATURE
}

\section{CUIDADOS DE ENFERMERÍA A LÓS NIÑOS CON CÁNCER: UNA REVISIÓN INTEGRADORA DE LA LITERATURA}

\author{
Thiago Privado da Silva \\ Joséte Luzia Leite \\ Nereida Lucia Palko dos Santos \\ Ítalo Rodolfo Silva \\ Ana Carolina Abeid Mendonça \\ Maria José Carvalho Santos \\ Laura Johanson da Silva
}

Doi:10.5902/217976926918

RESUMO: Objetivo: revisar a produção científica acerca dos cuidados de enfermagem à criança com câncer. Método: estudo bibliográfico, descritivo usando o método da revisão integrativa da literatura. Os dados foram coletados em janeiro de 2013 utilizando as bases de dados Literatura Latino-Americana e do Caribe em Ciências da Saúde, Bases de Dados de Enfermagem e Índice Bibliográfico Espanhol de Ciências da Saúde, em conformidade com critérios de inclusão previamente estabelecidos. Resultados: a amostra final foi constituída por dez estudos. 0 cuidado de enfermagem à criança com câncer é investigado sob várias perspectivas prevalecendo pesquisas no contexto hospitalar. Houve predominância de estudos descritivos, exploratórios de abordagem qualitativa. Os estudos ressaltam a necessidade de inserir a família nos planos de cuidados do enfermeiro. Conclusão: o estudo evidencia a necessidade de capacitação profissional e apoio psicológico aos profissionais que atuam no cuidado a esta clientela e valorização dos aspectos subjetivos nos cuidados.

Descritores: Enfermagem pediátrica; Enfermagem oncológica; Cuidados de enfermagem; Neoplasias.

ABSTRACT: Objective: to review the scientific production related to the nursing cares to the child with cancer. Method: bibliographic study, descriptive uses the integrative review method of the literature. The data was collect in January of 2013 utilizing as data's base Latin American and Caribbean Health Sciences Literature, Nursing Database and Bibliographic Index on Health Sciences from Spain, in accordance with the inclusion criteria previously established. Results: The final sample was constituted by ten studies. The nursing care to the child with cancer is investigated from many perspectives prevailing research in hospital setting. There was a predominance of descriptive, exploratory studies of qualitative approach. The studies underscore the need to put the family in nursing care plans. Conclusion: the study evidences the need of capacitation professional and psychological supporting to the nurse acting of this clientele and appreciation of the subjective aspects of care.

Descriptors: Pediatric nursing; Oncologic nursing; Nursing care; Neoplasms.

RESUMEN: Objetivo: revisar la producción científica sobre de los cuidados de enfermería a los niños con cáncer. Método: estudio bibliográfico, descriptivo utilizando el método de 


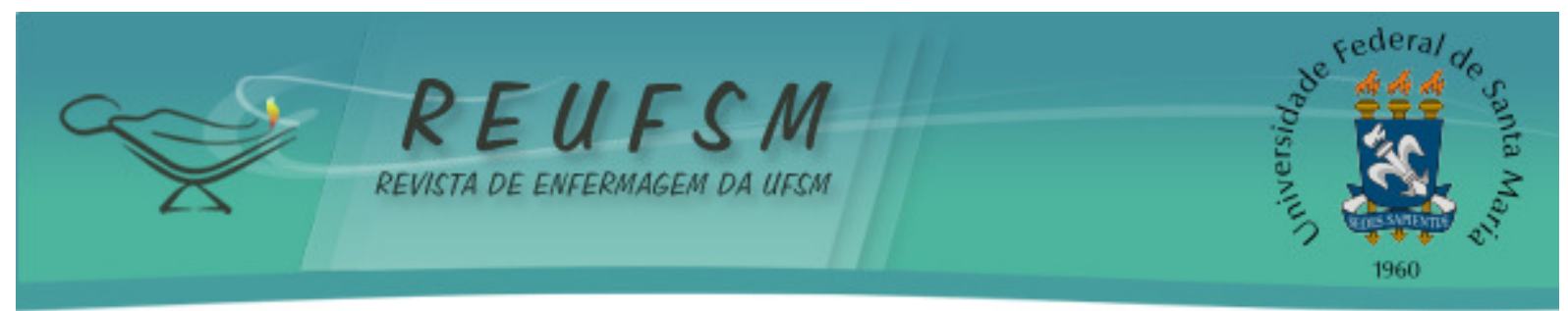

revisión integradora de la literatura. Los datos han sido recolectados en enero de 2013 utilizando bases de datos Literatura Latinoamericana y del Caribe em Ciencias de La Salud, Bases de Datos de Enfermería e Índice Bibliográfico Español em Ciencias de La Salud en conformidad con los criterios de inclusión establecidos previamente. Resultados: La muestra final fue compuesta por diez estudios. Los cuidados de enfermería a los niños con cáncer es investigado a través de diversas perspectivas prevaleciendo pesquisas en el ámbito hospitalario. Hubo un predominio de estudios descriptivos, exploratorios y de enfoque cualitativo. Los estudios resaltan la necesidad de poner a la familia en los planes de atención de enfermería. Conclusión: el estudio evidencia la necesidad de capacitación profesional y apoyo psicológico al enfermero que trabaja en el cuidado de la clientela y valoración de los aspectos subjetivos en la atención.

Descriptores: Enfermería Pediátrica; Enfermería oncológica; Cuidados de enfermería; Neoplasias.

\section{INTRODUÇÃO}

A Enfermagem é uma arte e ciência que requer do enfermeiro uma compreensão e aplicação de conhecimento e técnicas específicas com vistas a possibilitar tudo aquilo que o paciente necessita para realizar-se como ser independente, total e completo. ${ }^{1}$ Nesse sentido, a atuação deste profissional na oncologia pediátrica demanda além do conhecimento técnico e científico, afetividade na oferta do cuidado à criança e à família visando a promoção da saúde, qualidade de vida, conforto e bem-estar dos mesmos. Para tanto, o enfermeiro deve estar atento às singularidades e particularidades da criança e da família que se encontram sob seus cuidados, para assim, agir de maneira consciente, reflexiva e crítica no atendimento de suas necessidades.

Nesse sentido ressalta-se a imperiosidade de um gerenciamento flexível e criativo do cuidado com vistas a minimizar o sofrimento e dificuldades que permeiam o tratamento da criança com câncer. No estudo entende-se o gerenciamento do cuidado de enfermagem como expressão chave do trabalho do enfermeiro, momento no qual a articulação entre as dimensões gerencial e assistencial visam atender as necessidades de cuidado da criança, da equipe de enfermagem e da instituição. ${ }^{2}$

0 cuidado à criança com câncer é complexo, pois envolve múltiplos aspectos. ${ }^{3}$ Nesse contexto é importante que o enfermeiro articule saberes e práticas, busque aperfeiçoamento em oncologia e tenha habilidades para gerenciar o cuidado de enfermagem. ${ }^{3}$ Este, quando realizado considerando as tecnologias em saúde, é capaz de resolver problemas, estreitar vínculos e favorecer a co-responsabilidade nas relações de cuidado. ${ }^{4}$

O câncer infantil pode ser compreendido como um grupo de doenças crônicas não transmissíveis que atinge crianças e adolescentes de 0 a 19 anos que tem em comum o aparecimento de células modificadas que se multiplicam rápido e desordenadamente em algum órgão interferindo no seu funcionamento. Na infância os tipos mais comuns de câncer são: as leucemias, os tumores cerebrais, os linfomas, os tumores dos rins, os sarcomas entre outros. ${ }^{5}$ Nessa conjuntura o câncer infantil representa entre $1 \%$ a 3\% de todos os tumores malignos na maioria da população. ${ }^{5}$

As últimas informações disponíveis concernentes à mortalidade revelam que no ano de 2009, os óbitos por neoplasias na faixa etária de 1 a 19 anos, encontravam-se entre as dez primeiras causas de morte no Brasil e a partir de cinco anos, a morte por câncer corresponde a primeira causa de morte por doença em meninos e meninas. ${ }^{6}$ Por outro lado, ressalta-se que $70 \%$ das crianças diagnosticadas com câncer têm chance de cura quando o diagnóstico ocorre precocemente e o tratamento é realizado em centros especializados 


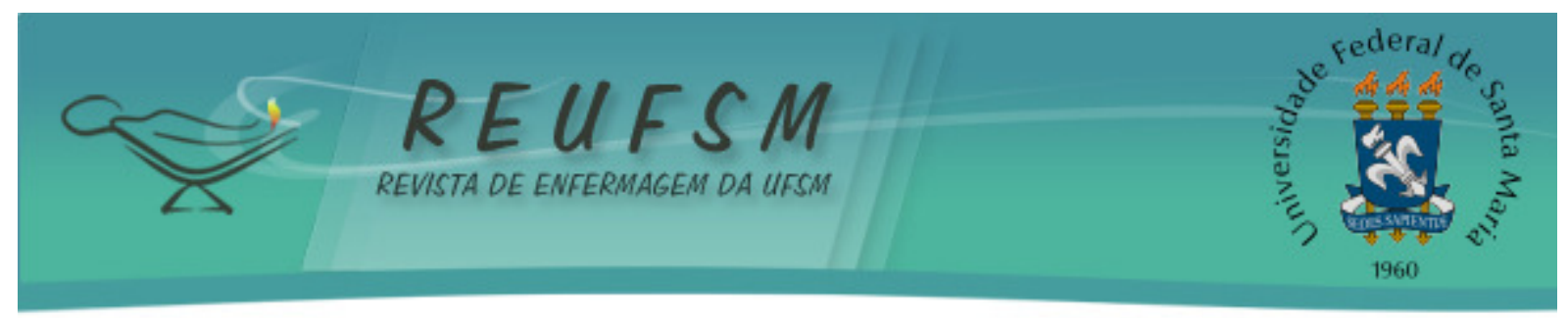

onde são evidentes os progressos alcançados em virtude do desenvolvimento científico e tecnológico. ${ }^{7}$

A este respeito o diagnóstico precoce do câncer conta com a ajuda da família, a qual deve procurar assistência médica sempre quando perceber qualquer alteração no corpo da criança. Isso tende a aumentar as chances de cura e a tornar o tratamento menos traumático para a mesma. Quando diagnosticada a doença, é preciso que a equipe de saúde tenha a consciência da importante presença e participação da família em todo o tratamento do infante.

Assim, a família precisa estar inserida no processo de cuidar da criança. Para tanto, precisa conhecer a patologia, suas manifestações e implicações, aliada a necessidade de ter sua habilidade de cuidar trabalhada, a fim de melhor assisti-la na perspectiva terapêutica proposta. Desse modo ela poderá desenvolver um cuidado cotidiano de qualidade e com autonomia, prevenindo agravos à saúde do infante. ${ }^{8}$ Ao enfermeiro nesse contexto cabe reconhecer as potencialidades do familiar no contexto e planejamento do cuidado, valorizando-o como unidade de cuidado, carecendo orientar e estabelecer estratégias que facilitem o aprendizado e o desenvolvimento do planejamento pactuado para o cuidado à criança.

Diante do exposto questiona-se: Em quais vertentes o cuidado à criança com câncer vem sendo estudada? Em quais contextos? Quais metodologias estão sendo empregadas nos estudos? Estes questionamentos permitiram construir a questão norteadora do estudo: qual o conhecimento científico que tem sido produzido na literatura sobre os cuidados diretos e/ou indiretos à criança com câncer? Portanto, objetiva-se revisar a produção científica sobre os cuidados de enfermagem à criança com câncer para uma posterior discussão dos resultados. O estudo se justifica enquanto proposta da Política Nacional de Atenção Oncológica ${ }^{9}$ que estimula a pesquisa na Atenção Oncológica, visando a qualificação da assistência e a promoção da educação permanente aos profissionais de saúde segundo os princípios da integralidade e humanização. Encontra-se também dentro das necessidades da Agência Nacional de Prioridades de Pesquisa em Saúde que incentiva pesquisas que versam sobre práticas terapêuticas voltadas à prevenção, reabilitação e qualidade de vida da criança. ${ }^{10}$

A relevância do estudo repousa na possibilidade de ampliar o conhecimento acerca da temática em tela, tendo como foco a atuação do enfermeiro no contexto dos cuidados em saúde. Aliada a esta perspectiva é possível que o estudo evidencie lacunas sobre a temática sugerindo a realização de novas pesquisas em oncologia pediátrica.

\section{MÉTODO}

Estudo bibliográfico de caráter descritivo utilizando o método da revisão integrativa da literatura para coleta e análise dos dados. A revisão integrativa da literatura é um método de pesquisa que possibilita a síntese do estado do conhecimento de uma determinada temática, identificando lacunas existentes para sugestões de novos estudos e perspectivas da temática estudada. ${ }^{11}$ Para tanto, foram observadas as seguintes etapas: elaboração da pergunta norteadora; busca ou amostragem na literatura; coleta de dados; análise crítica dos estudos incluídos; discussão dos resultados e apresentação da revisão/síntese do conhecimento. ${ }^{12}$

Os dados foram coletados em janeiro de 2013 e para o levantamento dos mesmos foi utilizada como bases de dados o LILACS (Literatura Latino-Americana e do Caribe em Ciências da Saúde), BDENF (Bases de Dados de Enfermagem) e o IBECS (Índice Bibliográfico Espanhol de Ciências de Saúde). Como estratégias de busca, foram utilizados descritores de assunto contidos nos Descritores em Ciências da Saúde - DeCS, a saber: Enfermagem 


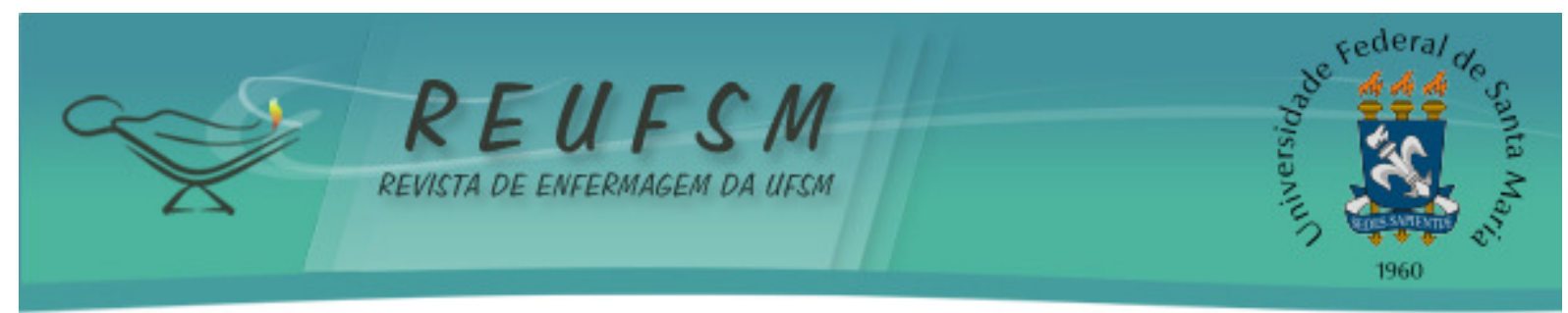

Pediátrica, Cuidados de Enfermagem, Enfermagem Oncológica, Neoplasias. Os descritores foram combinados nas seguintes formas: Neoplasias AND Cuidados de Enfermagem; Neoplasias AND Enfermagem Oncológica; Enfermagem Oncológica AND Enfermagem Pediátrica. Delineou-se como critérios de elegibilidade: estudos completos publicados com resumos disponíveis em inglês, português ou espanhol, nos últimos dez anos, apresentando o cuidado de enfermagem direto e/ou indireto à criança com câncer como objeto de estudo. Considera-se o intervalo de tempo amplo e suficiente para captar artigos atualizados sobre a temática.

Almejando garantir adequada captação e organização dos dados foi elaborado um instrumento contendo as seguintes variáveis: identificação do estudo, categoria profissional dos autores, ano de publicação, características metodológicas, contextos e perspectivas do cuidado de enfermagem, principais resultados e contribuições para enfermagem. Os estudos foram analisados criticamente por meio de leitura na íntegra. Após análise, foi realizada uma síntese dos estudos selecionados sendo organizados em temas, observando as suas confluências e divergências gerando duas categorias: aspectos gerenciais do enfermeiro no cuidado à criança com câncer; desafios do enfermeiro na atenção paliativa oncológica pediátrica.

\section{RESULTADOS E DISCUSSÕES}

O corpus literário foi constituído por dez estudos os quais atenderam aos critérios de inclusão previamente estabelecidos, sendo cinco da BDENF, quatro do LILACS e um do IBECS. A apresentação dos dados e discussão dos resultados é feita de forma descritiva e comparativa apoiando-se em literatura pertinente do assunto visando atender ao objetivo do estudo.

Concernente à categoria profissional dos autores e haja vista que o objeto de estudo investigado é pertencente à área da enfermagem, ressalta-se que todas as publicações selecionadas são de autoria de enfermeiros sendo que em dois estudos ${ }^{13-14}$ observou-se a participação de graduandos em Enfermagem. A este respeito ressalta-se que - enfermeiro desde a sua formação deve receber incentivo para desenvolvimento, participação e aplicação de pesquisas como também outras formas de produção de conhecimento visando à qualificação da prática profissional. ${ }^{15}$

Considerando a variável titulação Strictu Sensu, dez enfermeiros são Mestres em Enfermagem e nove enfermeiros foram identificados com título de Doutor em Enfermagem. Cabe ressaltar que a enfermagem brasileira enquanto profissão social vem trilhando por caminhos desafiadores visando consolidar-se como ciência, tecnologia e informação contando com 51 Programas de Pós-Graduação em Enfermagem no Brasil.16 Relativamente a Pós-Graduação Latu Sensu, identificou-se um enfermeiro especialista em Sistema Público de Saúde, um enfermeiro especialista em Violência Doméstica Contra Crianças e Adolescentes e outro especialista em Pediatria e Neonatologia.

Quanto ao ano de publicação, um estudo foi publicado no ano de $2004^{17}$, dois estudos foram publicados em $2005^{14^{3}, 18}$, um em $2007^{19}$ e $2009^{13}$, dois publicados em $2010^{3,20}$ um em $2011^{21}$ e dois em 2012. ${ }^{22-23}$ No delineamento do estudo, verificou-se um relato de experiência $^{3}$, uma pesquisa documental do tipo descritiva e retrospectiva ${ }^{21}$, sete estudos descritivos exploratórios ${ }^{13-14,17,19-20,22-23}$ e um estudo de revisão de literatura. ${ }^{18}$ Os resultados evidenciam que há pouco estudo de revisão acerca da temática. Os estudos de revisões têm importante papel na prática baseada em evidência na enfermagem. ${ }^{12} \mathrm{~A}$ revisão sistemática e meta-análise configuram-se como métodos de revisão mais utilizados, porém embora importantes, não são capazes de contemplar importantes questões relacionadas aos aspectos do cuidado e ao impacto da doença ou do tratamento. ${ }^{12}$ 


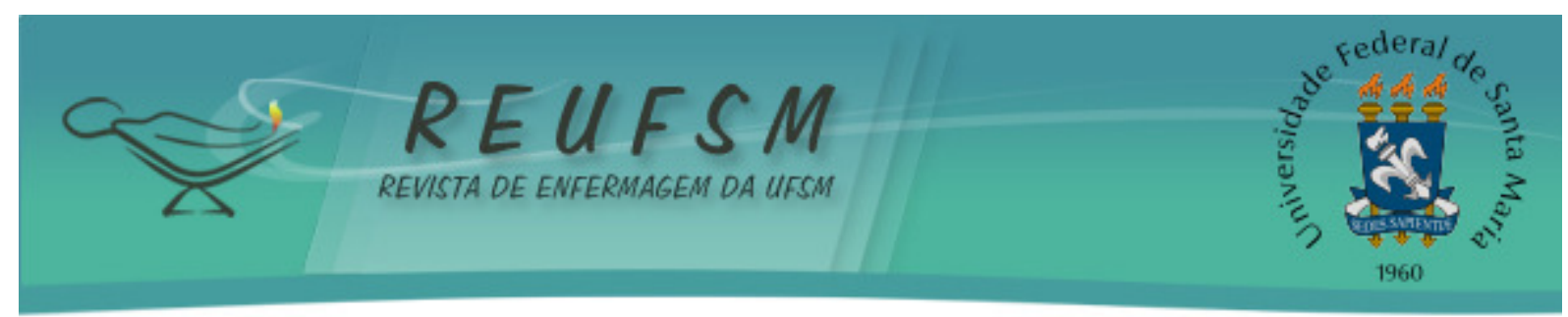

No que se refere às abordagens utilizadas, observou-se uma predominância de sete estudos de abordagem qualitativa ${ }^{13-14,17,19-20,22-23}$ e um estudo de abordagem quantitativa. ${ }^{21} \mathrm{~A}$ abordagem qualitativa possibilita a produção de conhecimentos sobre fenômenos subjetivos e se apresenta como foco de interesse da Enfermagem. ${ }^{24}$ Sob esta perspectiva, a valorização da abordagem qualitativa pelos pesquisadores da área da enfermagem, reafirma que estes profissionais vêm buscando na sua prática profissional caminhos epistemológicos para ampliar as possibilidades de olhar o ser humano como um todo sem perder de vista as especificidades, evitando a fragmentação do mesmo. ${ }^{25}$

No que tange aos contextos onde os estudos foram desenvolvidos, sete foram realizados em unidades de internação hospitalar ${ }^{13-14,17,20-21,23}$, dois estudos no ambulatório de quimioterapia pediátrica, ${ }^{3,22}$ e outro na unidade de terapia intensiva pediátrica. ${ }^{22} \mathrm{Em}$ geral, o cuidado do enfermeiro à criança com câncer pode acontecer tanto a nível hospitalar quanto a nível ambulatorial, pois são nestes cenários que se concentram esta clientela. Porém, conforme evidenciado no estudo existe um considerável interesse de estudos em unidades de internação hospitalar o que sugere a realização de estudos em outras unidades. Dos estudos analisados seis foram desenvolvidos na região sudeste, nos estados do Rio de Janeiro ${ }^{3,13,19,23}$ e São Paulo ${ }^{14,17}$, dois na região sul, no estado do Rio Grande do Sul ${ }^{21-22}$ e um na região Nordeste, no estado da Paraíba. ${ }^{20}$

Quanto às vertentes estudadas, identificou-se um estudo relacionado à prática do gerenciamento do cuidado à criança com câncer ${ }^{3}$; duas investigações sobre a atuação do enfermeiro no manejo da dor da criança hospitalizada ${ }^{17,21}$; a relação da prática e a busca pela produção do conhecimento no cuidado à criança com câncer ${ }^{20}$; dois acerca da percepção do enfermeiro diante da criança com câncer sob cuidados paliativos ${ }^{13,19}$, um sobre a percepção e sentimentos do enfermeiro no cuidado à criança com câncer ${ }^{14}$, uma revisão de literatura na área da oncologia pediátrica ${ }^{18}$ e dois estudos sobre o significado para equipe de enfermagem de cuidar de uma criança que tem doença oncológica avançada. ${ }^{22-23}$

\section{Aspectos gerenciais do enfermeiro no cuidado à criança com câncer}

O cuidado à criança com câncer requer do enfermeiro o desenvolvimento de habilidades e competências gerenciais para que possa atender as complexas necessidades da criança e sua família. Para tanto, torna-se imperioso que o enfermeiro tenha consciência do seu importante papel na liderança da equipe de enfermagem e que esteja atento não somente as necessidades da criança e sua família, como também, as necessidades de sua própria equipe.

Em um estudo ${ }^{3}$, evidencia-se a necessidade de um gerenciamento do cuidado dinâmico à criança com câncer e que o mesmo seja realizado de acordo com as situações vivenciadas. Em vista disso, o enfermeiro deve utilizar capacidade de julgamento, decisão, reflexão, integração, intencionalidade, criatividade e utilização do conhecimento científico. 0 estudo ressalta a valorização das relações interpessoais buscando desfazer hierarquias e ainda a liderança como fundamental para o enfermeiro nas atividades assistenciais e gerenciais.

0 enfermeiro líder é capaz de analisar criticamente a situação, identificar problemas, tomar decisões, planejar e implementar cuidados, alocar profissionais da equipe de enfermagem, motivar os profissionais da equipe na realização das atividades, definir a estrutura de trabalho de sua equipe e instituição, influenciar na administração, na educação, na pesquisa, no processo decisório, no aprimoramento e autonomia de seus colaboradores possibilitando assistência integral ao paciente. ${ }^{26-27}$ A liderança envolve compromisso, responsabilidade, empatia, habilidade para tomada de decisões, 


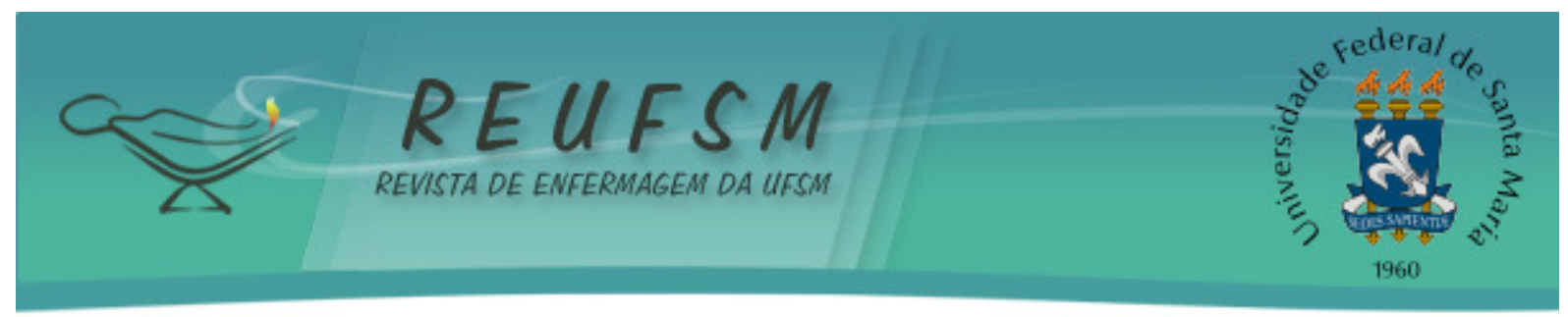

comunicação e gerenciamento de forma efetiva e eficaz. ${ }^{15}$ Desse modo, a liderança influencia positivamente os cuidados de enfermagem à criança com câncer e se apresenta como subsídio para uma gerência efetiva e eficaz.

0 enfermeiro apresenta-se como profissional vital na equipe de saúde em unidade de oncologia pediátrica. ${ }^{3} \mathrm{Na}$ sala de quimioterapia ambulatorial, por exemplo, ele detém a responsabilidade em executar atividades assistenciais, gerencias e educativas entre as quais estão orientação aos pais e às crianças sobre a patologia, o tratamento, cuidados necessários, complicações, sintomatologia de doenças oportunistas, educação permanente à equipe sobre protocolos quimioterápicos, especificidades assistenciais relacionadas ao diagnóstico médico e de enfermagem, atualização quanto ao uso de novas tecnologias de cuidado, capacitação dos profissionais para manipulação de cateter, atuação junto ao Controle de Infecção Hospitalar prevenindo infecção cruzada e doenças transmissíveis em geral, entre outras atividades que lhe são cabíveis. ${ }^{3}$

Consta em um estudo18 que os cuidados de enfermagem na oncologia pediátrica têm como objetivo reduzir a ansiedade, estado de depressão e o medo da criança e sua família; promover e incentivar a expressão dos sentimentos; incentivar a adesão, comunicação e melhorar o relacionamento; ajudar na organização familiar para uma melhor puericultura; reduzir e evitar riscos de infecção, hemorragia; orientar a criança e família; evitar dor, desidratação; prevenir náuseas e vômitos; manter e melhorar estado nutricional; estimular apetite; ajudar na adaptação da criança. Todas essas metas se tornam viáveis quando o gerenciamento do cuidado de enfermagem é realizado de forma participativa e efetiva.

A despeito desta prática os enfermeiros da oncologia pediátrica segundo um estudo14 devem deixar a tradicional forma de pensar que doam cuidados básicos e assumir a posição de educadores e apoiadores da família da criança. Dito isso é importante ressaltar que a família, em especial os pais, também vivencia a internação da criança e sofre com a mesma no percurso de todo o tratamento. A família se apresenta ora como cuidadora e ora necessitando dos cuidados do enfermeiro. Sob esta perspectiva a literatura, em especial da enfermagem, evidencia uma impossibilidade em oferecer cuidado de qualidade à criança com condição crônica sem inserir a família no plano de cuidados. Logo, é preciso conhecê-la no seu cotidiano buscando identificar elementos contextuais relevantes para o cuidado de enfermagem. ${ }^{8}$

É essencial que a criança tenha apoio dos pais ou familiares para enfrentar as dificuldades inerentes ao tratamento. ${ }^{3}$ A criança encontra nos pais e em seus familiares o suporte necessário para o enfrentamento da doença e do tratamento, favorecendo a adaptação e adesão à terapêutica selecionada. Nesse percurso a literatura28 evidencia que ela acaba revelando-se mais amadurecida ficando psicologicamente estável, colaborativa, com o vocabulário mais rico ao utilizar termos médicos configurando-se como agente participante do seu próprio cuidado. Assim, é de grande relevância o papel que a equipe de enfermagem assume no lidar com os pais da criança, tendo que aliar sensibilidade ao conhecimento teórico com a finalidade de oferecer um cuidado qualificado e humanizado. ${ }^{29}$ Portanto, ao gerenciar o cuidado é importante que o enfermeiro reconheça a relevante participação da família no tratamento da criança.

Um estudo ${ }^{14}$ aponta que no gerenciamento do cuidado de enfermagem é fundamental que o enfermeiro saiba orientar sua equipe a oferecer cuidados de forma humanizada, entendendo e respeitando a família em seu contexto e complexidade. Segundo o mesmo estudo, como o cuidado não se limita somente a realização de procedimentos técnicos, os enfermeiros tendem a direcionar suas ações para fornecer suporte emocional e psicológico à criança e à família. 


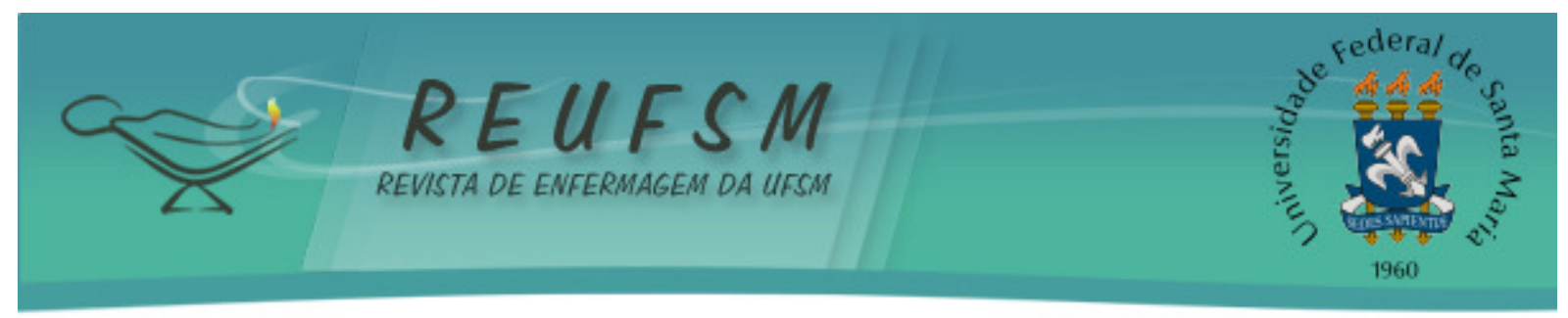

Desafios do enfermeiro na atenção paliativa oncológica pediátrica

No que se refere ao cuidado à criança com câncer nem sempre ela apresenta possibilidades de cura necessitando de uma modalidade de assistência baseada em cuidados paliativos. 0 cuidado paliativo à criança é entendido como uma abordagem de cuidado integral e ativo, o qual engloba elementos físico, emocional, social e espiritual, centrando-se no aumento da qualidade de vida para a criança e suporte para a família mediante controle de sintomas angustiantes, cuidado durante a morte e no luto. ${ }^{30}$ Nesse contexto cabe aos profissionais da equipe de enfermagem a responsabilidade de resgatar autoestima, conforto e individualidade do paciente e sua família. ${ }^{31}$

A atuação do enfermeiro na atenção paliativa oncológica pediátrica é acompanhada por inúmeros desafios os quais influenciam sobremaneira o modo de gerenciar o cuidado de enfermagem. Um dos grandes desafios para o enfermeiro e demais profissionais da equipe de saúde é proporcionar qualidade de vida à criança com câncer em cuidados paliativos. Isso exige primeiramente uma mudança paradigmática para a compreensão de que as ações direcionadas à criança na atenção paliativa oncológica não visam à cura, mas a qualidade de vida durante o viver/morrer da criança. É importante que o profissional saiba administrar essa situação, pois a morte não pode ser antecipada nem postergada, mas devidamente controlada em suas circunstâncias. ${ }^{19}$

Diante do exposto, um estudo17 aponta que no cuidado ao paciente fora de possibilidade de cura o controle da dor é destacado como uma das prioridades da assistência. Os resultados desse estudo revelam que os profissionais reconhecem o critério de avaliação com base nos aspectos comportamentais e fisiológicos descritos na literatura obedecendo as fases de crescimento e desenvolvimento da mesma. No entanto, alguns profissionais relataram uma maior dificuldade na avaliação da dor quando a criança não pode se expressar verbalmente. Esse resultado também foi encontrado em outro estudo ${ }^{21}$ que revelou problemas na localização e avaliação da dor em lactentes e pré-escolares que se expressam por meio do choro e pouco conseguem descrever a dor que sentem.

Entre os fatores que podem facilitar na avaliação da intensidade da dor, está a presença da mãe, sobretudo quando a criança é menor e ainda não verbaliza seus sentimentos e um outro fator, é a empatia que deve existir entre a criança e o profissional. ${ }^{17}$ Ao desenvolver uma relação empática o profissional tem a possibilidade de conhecer os problemas do outro o mais claramente possível favorecendo a resolução do problema a partir do ponto de vista da pessoa que se encontra sob seus cuidados. ${ }^{32}$

No que se refere ao manejo da dor, um estudo21 realizado com base nos registros da enfermagem constatou que o uso do medicamento seguindo a prescrição médica foi a escolha predominante pela equipe de enfermagem e destaca as três medicações mais utilizadas: paracetamol $(42,2 \%)$, morfina $(25 \%)$ e codeína $(6,9 \%)$. No que tange às estratégias não medicamentosas, identificou-se quatro registros de condutas: colo da mãe, troca de decúbito, uso da bolsa de água quente e a mamadeira.

Outro aspecto relacionado à atuação do enfermeiro na atenção paliativa oncológica se trata do significado desse profissional no cuidado à criança em risco iminente de morte. Nessa situação um estudo ${ }^{13}$ mostra que esses profissionais sofrem devido às dificuldades de encarar o sofrimento da criança e sua família. A angústia e o fracasso foram citados como sentimentos referentes à percepção dos enfermeiros diante da criança com câncer em cuidados paliativos.

0 cuidado à criança com câncer é desgastante e triste. ${ }^{22}$ Nas relações de cuidado à criança com câncer é inevitável não se envolver ainda que se estabeleça limites para o não envolvimento excessivo. A este respeito um estudo ${ }^{22}$ evidencia que o enfermeiro no cotidiano do cuidado a esta clientela expressa um esgotamento emocional e físico 


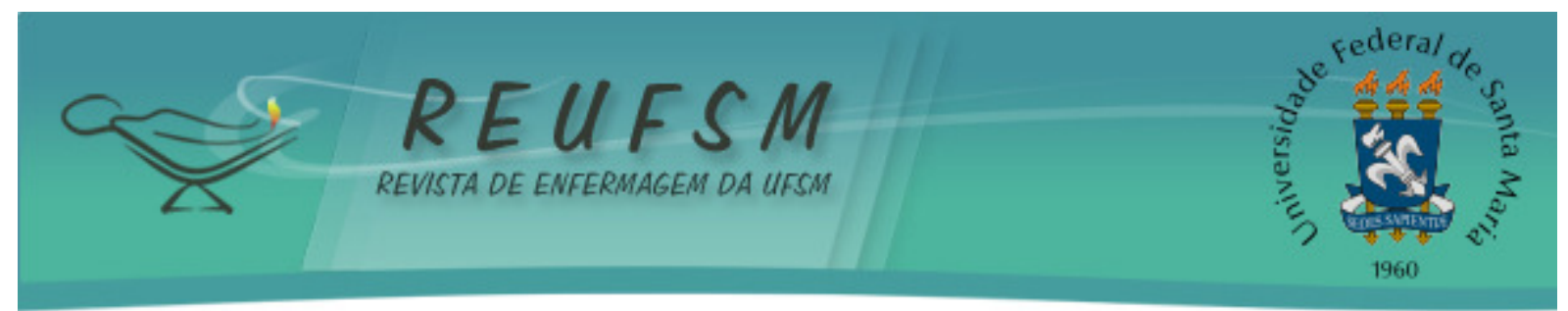

decorrente do envolvimento com a criança e sua família, do avanço da doença e da necessidade de ter que estar preparado para lidar com os entes que vão ao encontro da criança. Ao cuidar de uma criança sem possibilidade de cura, o enfermeiro desenvolve uma ação social baseada na compreensão do contexto familiar buscando se aproximar dos familiares para confortá-los. ${ }^{23}$

A falta de recursos para o profissional trabalhar a sua dimensão psicológica resulta em traumas e barreiras gerando conflitos e ansiedades que podem influenciar negativamente a relação entre equipe e família. ${ }^{14}$ Os desafios e dificuldades aqui apresentados revelam que os profissionais que atuam nesse setor necessitam de um apoio psicológico e constante capacitação para se sentirem acolhidos e motivados a continuarem nesse ambiente de cuidado.

A despeito deste fato são necessárias mudanças na estrutura organizacional hospitalar favorecendo momentos nos quais os profissionais possam se sentir amparados e seguros, reciclem seus conhecimentos sobre a doença e atividades nesta área e tenham informações das crianças que cuidam. ${ }^{14}$

\section{CONCLUSÕES}

O estudo revela que o cuidado de enfermagem à criança com câncer é uma ação social complexa permeada por incertezas e por uma relação dialógica vida e morte, ordem e desordem. Trata-se de um cuidado onde o conhecimento técnico e científico e o apoio psicológico para controle das emoções e possivelmente para o alívio do sofrimento revelam-se como necessários aos enfermeiros e sua equipe para uma prática humanizada e qualificada. O conhecimento disponível na literatura acerca do cuidado de enfermagem à criança com câncer revela que essa temática é estudada sob várias perspectivas contribuindo para a ampliação do conhecimento na área da oncologia pediátrica. A abordagem qualitativa foi a mais utilizada nos estudos revelando o interesse dos pesquisadores em realizar análises compreensivas acerca da vivência e experiência do enfermeiro no cuidado a esta clientela.

0 gerenciamento do cuidado de enfermagem se apresentou como prática necessária para o planejamento, organização, supervisão e implementação dos cuidados de enfermagem. No aspecto gerencial o exercício da liderança oferece subsídios para o enfermeiro gerenciar a equipe motivando seus liderados a realizarem suas atividades com competência e eficácia. 0 enfermeiro revelou-se como profissional vital na oncologia pediátrica e sua atuação não deve se dar de forma isolada, mas em consonância com o trabalho dos demais profissionais da equipe.

Foi possível identificar dificuldades dos profissionais em avaliar a intensidade da dor em crianças lactentes e pré-escolares. Observou-se que o tratamento medicamentoso foi a estratégia mais utilizada para o alívio da dor da criança com câncer. Além dessa, a literatura revelou o uso da mamadeira, colo da mãe, bolsa de água quente e troca de decúbito como estratégia não medicamentosa.

A promoção da qualidade de vida à criança em cuidados paliativos revelou-se como desafio para o enfermeiro e sua equipe. 0 apoio à família e a sua inserção nos planos de cuidado também se apresentam como desafio às competências do enfermeiro. Os estudos evidenciaram que os profissionais se sentem desgastados emocionalmente e fisicamente diante do sofrimento da criança e sua família. Revelaram se sentir tristes e angustiados com a situação da criança carecendo de apoio psicológico e capacitação para atuação nesse cenário. Enfatiza-se a necessidade de valorização dos aspectos subjetivos nas relações de cuidado e o desenvolvimento de habilidades e competências para lidar com os mesmos. 


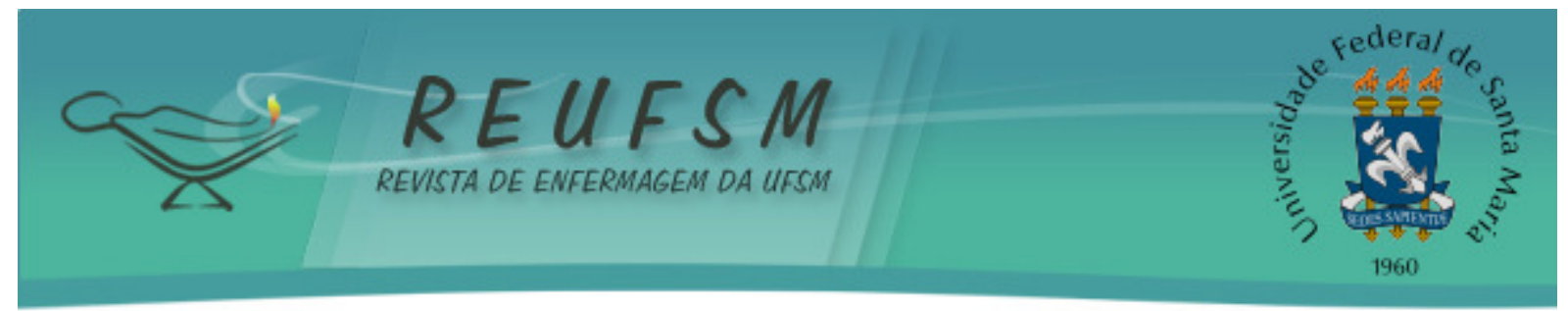

\section{REFERÊNCIAS}

1. Sol AG, Vázquez RF. Influencia de la gestión del cuidado en la calidad de la atención de salud. Rev Cuba Enferm [Internet]. 2010 abr-jun [acesso em 2012 mar 15];26(2):14-26. Disponível em: http://bvs.sld.cu/revistas/enf/vol26_2_10/enf03210.htm.

2. Hausmann $M$, Peduzzi M. A articulação entre as dimensões gerencial e assistencial do processo de trabalho do enfermeiro. Texto \& Contexto Enferm. 2009;18(2):258-65.

3. Gomes IP, Reis PED, Collet N. Management of nursing's care in pediatric ambulatory chemotherapy unit. Rev Enferm UFPE on line [Internet]. 2010 Apr [acesso em 2012 mar 24];4(2):510-16.

Disponível

em:

http://www.ufpe.br/revistaenfermagem/index.php/revista/article/view/646/pdf_42.

4. Rossi FQ, Silva MAD. Fundamentos para processos gerenciais na prática do cuidado. Rev Esc Enferm USP [Internet]. 2005 [acesso em 2013 jan 23];39(4):460-8. Disponível em: http://www.scielo.br/pdf/reeusp/v39n4/12.pdf.

5. Associação Capixaba contra o Câncer Infantil. Sobre o câncer [acesso em 2012 fev 26]. Disponível em: http://www.acacci.org.br/info.htm.

6. Brasil. Ministério da Saúde. Instituo Nacional de Câncer - INCA. Estimativa 2012: incidência de câncer no Brasil [Internet]. 2011 [acesso em 2012 fev 26]. Disponível em: http://www.inca.gov.br/estimativa/2012.pdf.

7. Brasil. Ministério da Saúde. Secretaria de Atenção à Saúde. Instituto Nacional de Câncer. Particularidades do câncer infantil. Brasília: Ministério da Saúde; 2008.

8. Araújo YB, Collet N, Moura FM, Nóbrega RD. Conhecimento da família acerca da condição crônica na infância. Texto \& Contexto Enferm. 2009;18(3):498-505.

9. Brasil. Ministério da Saúde. Instituto Nacional do Câncer. Portaria $n^{\circ} 2.439 / \mathrm{GM}$, de 8 dezembro de 2005. Institui a Política Nacional de Atenção Oncológica: Promoção, Prevenção, Diagnóstico, Tratamento, Reabilitação e Cuidados Paliativos, a ser implantada em todas as unidades federadas, respeitadas as competências das três esferas de gestão. Diário Oficial da União, Brasília; 2005.

10. Brasil. Ministério da Saúde. Secretaria de Ciência, Tecnologias e Insumos Estratégicos. Departamento de Ciência e Tecnologia. Agenda Nacional de Prioridades de Pesquisa em Saúde, $2^{\mathrm{a}}$ ed. Brasília: Editora do Ministério da Saúde; 2008.

11. Polit DF, Beck CT. Using research in evidence-based nursing practice. In: Polit DF, Beck $\mathrm{CT}$, editors. Essentials of nursing research. Methods, appraisal and utilization. Philadelphia (USA): Lippincott Williams \& Wilkins; 2006. p.457-94.

12. Souza MT, Silva MD, Carvalho R. Revisão integrativa: o que é e como fazer. Einstein. 2010;8:102-6.

13. Avanci BS, Carolindo FM, Goés FGB, Netto NPC. Cuidados paliativos à criança oncológica na situação do viver/morrer: a ótica do cuidar em enfermagem. Esc Anna Nery Rev Enferm. 2009;13(4):708-16.

14. Paro D, Paro J, Ferreira DLM. O enfermeiro e o cuidar em oncologia pediátrica. Arq Ciênc Saúde. 2005;12(3):151-7.

15. Conselho Nacional de Educação. Câmara de Educação Superior. Resolução CNE/CES 3/2001. Diário Oficial da União, Brasília, 9 nov 2001. Seção 1, p.37. 


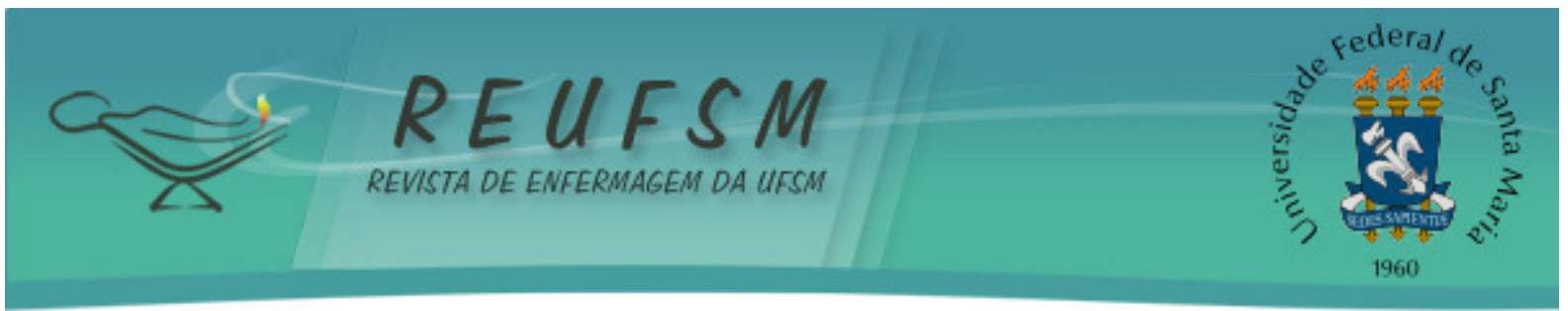

16. Erdmann AL. A importância da publicação científica. Rev Enferm UFSM [Internet]. 2011 maio-ago [acesso em 2013 mar 25];1(2). Disponível em: http://cascavel.ufsm.br/revistas/ojs2.2.2/index.php/reufsm/article/view/2995/1688

17. Menossi, MJ. A complexidade da dor da criança e do adolescente com câncer hospitalizados e as múltiplas dimensões do seu cuidar [dissertação]. Ribeirão Preto: Escola de Enfermagem de Ribeirão Preto; 2004. 118 p.

18. Palma C, Sepúlveda F. Atención de enfermeira em el niño com cáncer. Rev Ped Eletr [Internet]. 2005 ago [acesso em 2013 jan 25];2(2). Disponível em: http://www.revistapediatria.cl/vol2num2/10.htm.

19. Lopes VF, Silva JLL, Andrade M. The professional nursing perception about palliative cares to oncologic pediatric is of cure possibility patient: a study in phenomenological boarding of the relations human beings. Online Brazilian J Nurs [Internet]. 2007 [acesso em 2013 jan 25];6(3). Disponível em: http: //www.objnursing.uff.br/index.php/nursing/article/view/j.1676-

4285.2007.1155/268.

20. Amador DL, Gomes IP, Coutinho SED, Costa TNA, Collet N. Living pediatric oncology care and the quest for knowledge production. Rev Enferm UFPE on line [Internet]. 2010 Apr [acesso em 2012 mar 24];4(2):666-72. Disponível em: http://www.ufpe.br/revistaenfermagem/index.php/revista/article/view/851/pdf_53

21. Bueno PC, Neves ET, Rigon AG. O manejo da dor em crianças com câncer: contribuições para a enfermagem. Cogitare Enferm. 2011;16(2):226-31.

22. Especialidade do ser-profissional-de-enfermagem no mundo do cuidado à criança que tem câncer. Esc Anna Nery Rev Enf. 2012;16(3):493-9.

23. Monteiro ACM, Rodrigues BMRD, Pacheco STA. O enfermeiro e o cuidar da criança com câncer sem possibilidade de cura atual. Esc Anna Nery Rev Enferm. 2012;16(4):741-6.

24. Camelo, SHH. O trabalho em equipe na instituição hospitalar: uma revisão integrativa. Cogitare Enferm. 2011;16(4):734-40.

25. Crivaro ET, Almeida IS, Souza IEO. O cuidar humano: produção acadêmica de enfermagem. Rev Enferm UERJ. 2007;15(2):248-54.

26. Sousa LB, Barroso MGT. Reflexão sobre o cuidado como essência da liderança em enfermagem. Esc Anna Nery Rev Enferm. 2009;13(1):181-7.

27. Gelbcke FL, Souza LA, Dal Sasso G, Nascimento E, Bulb MBC. Liderança em ambientes de cuidados críticos: reflexões e desafios à Enfermagem Brasileira. Rev Bras Enferm. 2009;62(1):136-9.

28. Ribeiro CA, Ângelo M. O significado da hospitalização para a criança pré-escolar: um modelo teórico. Rev Esc Enferm USP [Internet]. 2005 [acesso em 2012 mar 19]; 39(4):391400. Disponível em: http://www.scielo.br/pdf/reeusp/v39n4/03.pdf.

29. Duarte MLC, Zanini LN, Nedel MNB. O cotidiano de pais de crianças com câncer e hospitalizadas. Rev Gaúcha Enferm. 2012;33(3):111-8.

30. Floriani CA. Cuidados paliativos no domicílio: desafios aos cuidados de crianças dependentes de tecnologias. J Pediat [Internet]. 2010 [acesso em 2012 mar 20];86(1):1520.

Disponível

em:

http://cascavel.ufsm.br/revistas/ojs2.2.2/index.php/reufsm/article/view/2995/1688 


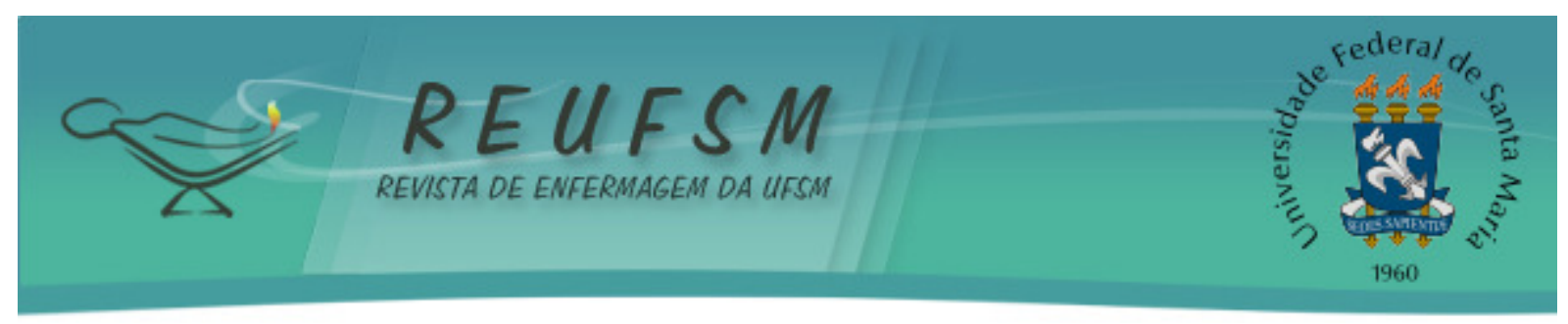

31. Silva AF, Issi HB, Motta MGC. A família da criança oncológica em cuidados paliativos: o olhar da equipe de enfermagem. Cienc Cuid Saúde [Internet]. 2011 [acesso em 2012 mar 20];10(4):820-7. Disponível em: http://eduemojs.uem.br/ojs/index.php/CiencCuidSaude/article/viewFile/18328/pdf.

32. Avanci RC, Furegato ARF, Scatina MCM, Pedrão LJ. Relação de ajuda enfermeiropaciente pós tentativa de suicídio. Rev Eletr Saúde Mental Alcool Drog [Internet]. 2009 fev [acesso em 2013 jan 25];5(1):1-15.

Data de recebimento: $20 / 10 / 12$

Data de aceite: 01/03/2013

Contato com autor responsável: Thiago Privado da Silva

E-mail: thiagopsilva87@gmail.com 